BMJ

Open

Gastroenterology

\title{
A pilot feasibility trial of cognitive- behavioural therapy for insomnia in people with inflammatory bowel disease
}

\author{
Jessica K Salwen-Deremer (1) , ${ }^{1,2}$ Michael T Smith, ${ }^{3}$ Kelly A Aschbrenner, ${ }^{2}$ \\ Hannah G Haskell, ${ }^{2}$ Brittany C Speed, ${ }^{2}$ Corey A Siegel ${ }^{1}$
}

\begin{abstract}
To cite: Salwen-Deremer JK, Smith MT, Aschbrenner KA, et al. A pilot feasibility trial of cognitive-behavioural therapy for insomnia in people with inflammatory bowel disease. BMJ Open Gastro 2021;8:e000805. doi:10.1136/ bmjgast-2021-000805

- Additional supplemental material is published online only. To view, please visit the journal online (http://dx.doi. org/10.1136/bmjgast-2021000805).
\end{abstract}

Received 14 October 2021 Accepted 13 December 2021

Check for updates

(C) Author(s) (or their employer(s)) 2021. Re-use permitted under CC BY-NC. No commercial re-use. See rights and permissions. Published by BMJ.

${ }^{1}$ Department of Medicine, Section of Gastroenterology \& Hepatology, DartmouthHitchcock Medical Center, Lebanon, NH, USA

'Department of Psychiatry, Dartmouth-Hitchcock Medical Center, Lebanon, NH, USA ${ }^{3}$ Psychiatry \& Behavioral Sciences, Johns Hopkins University School of Medicine, Baltimore, MD, USA

\section{Correspondence to} Dr Jessica K Salwen-Deremer; jessica.k.salwen-deremer@ hitchcock.org

\section{ABSTRACT}

Objective Poor sleep is common in inflammatory bowel disease (IBD), associated with worse overall disease course and predominantly attributable to insomnia. While cognitive-behavioural therapy for insomnia (CBT-I) is the recommended first-line treatment for chronic insomnia, it is untested in IBD. It is unclear if CBT-I will be as effective in this group given the extent of night-time symptoms people with IBD experience. Thus, we evaluated the feasibility and preliminary efficacy of CBT-I in IBD. Design We comprehensively assessed sleep in people with mild-to-moderately active IBD using questionnaires, daily diaries and actigraphy. People with significant insomnia symptoms were allocated to a single-arm, uncontrolled pilot feasibility study of gold-standard CBT-I treatment. They were then reassessed post-treatment. Results 20 participants with IBD completed a baseline assessment. 10 were experiencing insomnia and were allocated to CBT-I. All participants who were offered CBT-I elected to complete it, and all completed 5/5 sessions. Participants rated treatment acceptability highly and daily diary and actigraphy completion rates were $\geq 95 \%$. At baseline, participants with insomnia evidenced significantly worse sleep than participants without insomnia. Following CBT-I, participants reported significant improvements in diary and actigraphy measures of sleep continuity, dysfunctional sleep-related beliefs and IBD disease activity.

Conclusion CBT-I was feasible and acceptable and demonstrated a signal for efficacy in the treatment of insomnia in IBD. Importantly, the improvements in sleep continuity were consistent with the extant literature.

Future fully powered randomised controlled studies should evaluate whether treatment of insomnia can improve other aspects of IBD, including pain and inflammation.

Trial registration number NCT04132024.

\section{INTRODUCTION}

Poor sleep occurs in $\sim 75 \%$ of people with active inflammatory bowel disease (IBD) and prospectively predicts greater likelihood of symptom flares, surgery and/or hospitalisation. ${ }^{1-5}$ Sleep problems are also uniquely associated with poor quality of life in IBD and

\section{Summary box}

What is already known about this subject?

- Poor sleep is common in inflammatory bowel disease (IBD) and predicts worse disease course and severity. Insomnia is the most common sleep disorder and the recommended first-line treatment for insomnia is cognitive-behavioural therapy for insomnia (CBT-I). However, CBT-I is untested in IBD, and night-time symptoms could impact intervention uptake or efficacy.

What are the new findings?

- CBT-I is feasible, acceptable and preliminarily effective in IBD.

How might it impact on clinical practice in the foreseeable future?

- These findings support the use of CBT-I in IBD, highlight the importance of asking about sleep as part of clinical care, and pave the way for future randomised controlled trials.

people with IBD believe that sleep impacts and/or worsens gastrointestinal symptoms. ${ }^{367}$

Insomnia disorder, or prolonged difficulty falling asleep, staying asleep, or early morning awakening combined with daytime impairment, is the most common sleep disorder. $^{8}$ It has a population prevalence of $10 \%$, a fivefold increase in chronic medical and psychiatric conditions, and even higher rates in chronic pain populations..$^{9-14}$ In IBD, one longitudinal study $(\mathrm{N}=276)$ found that at baseline, $58 \%$ of people with IBD selfreported clinically significant insomnia symptoms. Insomnia was also the most common sleep problem. ${ }^{15}$ Another cross-sectional study $(\mathrm{N}=312)$ indicated that $50 \%$ of people with IBD likely meet criteria for insomnia disorder, with even higher rates in people with active IBD. ${ }^{16}$ 
Chronic insomnia disorder is typically understood from within a diathesis-stress framework, wherein predisposing (eg, delayed circadian rhythms; physiological reactivity), precipitating (eg, IBD flare) and perpetuating factors (eg, increasing time in bed (TIB) to make up for lost sleep) develop and maintain insomnia. ${ }^{17}$ IBD symptoms may lead to disruptions in sleep (ie, fragmentation), following which people engage in cognitive and behavioural patterns that are meant to compensate for lost sleep, but which instead maintain sleep problems, leading to insomnia.

Cognitive-behavioural therapy for insomnia (CBT-I) is the recommended first line treatment for chronic insomnia disorder by the American College of Physicians and the European Sleep Research Society, among others, due to its comparable effectiveness, low side effect profile and improved durability relative to pharmacotherapy. ${ }^{18-21}$ Gold-standard, multicomponent CBT-I involves 4-8 visits with a trained provider and improves sleep irrespective of medical and psychiatric comorbidities, age and sleep medication use. ${ }^{22-26}$ Remission rates for insomnia are typically around $60 \%$ and remotely delivered CBT-I is as effective as in-person treatment. ${ }^{27}{ }^{28}$ However, the sleep disruptions that occur in IBD are unique (eg, night-time awakenings for bowel movements, anxiety about nighttime incontinence, abdominal pain) and the feasibility and efficacy of CBT-I in IBD is untested.

As a preliminary step to understanding sleep in people with IBD, we comprehensively assessed sleep in people with mild-to-moderately active IBD using questionnaires, daily diaries and actigraphy. To explore feasibility and preliminary efficacy of CBT-I in IBD, we conducted a pilot trial of CBT-I in participants who reported significant insomnia symptoms.

\section{MATERIALS AND METHODS Trial design}

In phase I, patients with mild-to-moderate IBD symptoms were recruited for an observational study on sleep in IBD. All participants completed a comprehensive baseline assessment, including questionnaires, a daily diary of sleep and IBD symptoms, and objective assessment of their sleep continuity. Participants who completed this baseline assessment and whose questionnaire scores were indicative of significant insomnia symptoms were invited to participate in phase II, during which they received CBT-I (single-arm trial design). CBT-I was followed by assessment immediately and 1 month later.

\section{Participants}

Providers affiliated with the Dartmouth-Hitchcock IBD centre referred potentially eligible patients to this study over a 16-month period. IBD centre providers asked patients with mild-to-moderately active Crohn's disease (CD) or ulcerative colitis (UC) and no current restless leg syndrome or sleep apnea if they would be interested in receiving information from the research team about a study on sleep in IBD. Charts of interested patients were forwarded to the study team and a team member reached out by phone to provide information on the study design and aims. Interested patients were emailed a link to an electronic consent form.

Following informed consent, screening questionnaires were given to further determine eligibility. Inclusion criteria were (1) a documented diagnosis of CD or UC and (2) current mild-to-moderate IBD symptoms. To be included in the intervention study (phase II), participants were required to have an Insomnia Severity Index (ISI) score $\geq 8$ and report taking at least $30 \mathrm{~min}$ to fall asleep (sleep-onset latency, SOL) or spending at least 30 min awake during the night (wake after sleep onset, WASO). For both study phases, exclusion criteria were (1) a Patient Health Questionnaire-9 (PHQ-9) score $\geq 20$, (2) a Generalised Anxiety Disorder-7 (GAD-7) anxiety score $\geq 20$, (3) current alcohol or substance abuse, (4) current opioid use, (5) an unstable major psychiatric condition (via chart review), (6) probable or likely restless leg syndrome based on the Cambridge-Hopkins RLS questionnaire or (7) high risk for obstructive sleep apnea based on the STOP-Bang questionnaire combined with current symptoms.

Recruitment took place between October 2019 and January 2021, with a pause from March to April 2020 due to medical centre restrictions on in-person recruitment related to the COVID-19 pandemic. During that pause, we adapted our protocol to COVID-19 guidelines and made our entire study remote. Previously, the baseline visit and consent were performed in-person, although CBT-I was always designed to be delivered via telehealth. Data collection was complete by April 2021.

\section{Intervention}

A five-session CBT-I intervention was delivered individually via telehealth to enrolled participants over 7 weeks. Intervention also included three brief phone calls designed to make changes to participants' sleep schedules and problem-solve any issues with adherence. The intervention sessions and phone calls followed a detailed treatment protocol (table 1). The goals of CBT-I are to reduce behaviour and thought patterns that can perpetuate insomnia and improve overall quality of sleep. Importantly, our CBT-I intervention also included specific content about management of chronic pain as it relates to insomnia, which is different from traditional CBT-I. Prior to session 1, participants were given an electronic manual (39 pages) that included handouts to be referenced at each visit. This manual was based on standard guidelines and included content specific to IBD (eg, waking up at night with bowel movements). ${ }^{29}{ }^{30}$ Sleep restriction protocol specifics are detailed in online supplemental file. CBT-I sessions lasted $\sim 45 \mathrm{~min}$ and phone calls lasted $\sim 10 \mathrm{~min}$. Intervention was delivered by JS-D, clinical psychologist and diplomate in behavioural sleep medicine, or by BCS, a postdoctoral fellow in psychology at Dartmouth-Hitchcock trained and supervised by JS-D. 


\begin{tabular}{|c|c|}
\hline $\begin{array}{l}\text { Week } 1 \\
\text { Visit } 1\end{array}$ & $\begin{array}{l}\text { Biology of sleep } \\
\text { Overview of behavioural model of } \\
\text { insomnia } \\
\text { - Sleep restriction and stimulus control }\end{array}$ \\
\hline $\begin{array}{l}\text { Week } 2 \\
\text { Visit } 2\end{array}$ & $\begin{array}{l}\text { Review sleep diaries and adjust sleep } \\
\text { prescription } \\
\text { Sleep hygiene with goal setting } \\
\text { Gate control model of pain } \\
\text { Worry control procedures and relaxation } \\
\text { training }\end{array}$ \\
\hline $\begin{array}{l}\text { Week } 3 \\
\text { Phone call } 1\end{array}$ & $\begin{array}{l}\text { Adjust sleep prescription } \\
\text { Check in on progress with worry control } \\
\text { procedures and relaxation training }\end{array}$ \\
\hline $\begin{array}{l}\text { Week } 4 \\
\text { Visit } 3\end{array}$ & $\begin{array}{l}\text { Review sleep diaries and adjust sleep } \\
\text { prescription } \\
\text { Cognitive therapy: self-talk, cognitive } \\
\text { distortions, automatic thoughts, and how } \\
\text { thoughts contribute to insomnia and pain }\end{array}$ \\
\hline $\begin{array}{l}\text { Week } 5 \\
\text { Phone call } 2\end{array}$ & $\begin{array}{l}\text { Adjust sleep prescription } \\
\text { Check in on progress with thought log }\end{array}$ \\
\hline $\begin{array}{l}\text { Week } 6 \\
\text { Visit } 4\end{array}$ & $\begin{array}{l}\text { Review sleep diaries and adjust sleep } \\
\text { prescription } \\
\text { Cognitive therapy: disputing negative } \\
\text { thoughts }\end{array}$ \\
\hline $\begin{array}{l}\text { Week } 7 \\
\text { Phone call } 3\end{array}$ & $\begin{array}{l}\text { Adjust sleep prescription } \\
\text { Check in on progress with thought log }\end{array}$ \\
\hline $\begin{array}{l}\text { Week } 8 \\
\text { Visit } 5\end{array}$ & $\begin{array}{l}\text { Review sleep diaries and adjust sleep } \\
\text { prescription } \\
\text { Review treatment content and coping } \\
\text { strategies } \\
\text { Relapse prevention and maintenance } \\
\text { planning }\end{array}$ \\
\hline
\end{tabular}

CBT-I, cognitive-behavioural therapy for insomnia.

\section{Outcomes}

The feasibility of study procedures and the CBT-I intervention was assessed based on: (1) enrolment-toscreening ratio: number of patients enrolled out of the number screened, (2) adherence rate: number of participants who completed CBT-I sessions, (3) retention rate: number of participants who completed the post-CBT-I and follow-up assessments, (4) daily diary completion rate and (5) actigraphy completion rate. Acceptability of CBT-I was based on self-reported treatment acceptability and qualitative data on treatment experiences. Preliminary efficacy was assessed based on pre- to posttreatment change in (1) SOL, (2) WASO, (3) sleep efficiency (SE), (4) ISI, and (5) the Pittsburgh Sleep Quality Index (PSQI). We also explored the impact of CBT-I on Dysfunctional Beliefs and Attitudes about Sleep (DBAS) and IBD disease activity.

\section{Measures}

Daily diary

Participants completed an electronic daily diary during both phases of the trial and at the 1-month follow-up assessment. Morning diary items were based on a consensus sleep diary $^{31}$ and measures of SOL, WASO, total sleep time (TST) and TIB were abstracted from the diary. SE was calculated as TST/TIB $\times 100$. Mean SOL, WASO, TST and SE were computed for each week. Participants also completed an evening diary containing the PRO-3 and assessment of anxiety, stress and fatigue, rated from 0 (none) to 10 (worst imaginable).

\section{Actigraphy}

Actigraphs are wrist-worn, medical grade triaxial accelerometers that produce higher quality objective sleep data than commercial trackers. ${ }^{33}$ Based on standard guidelines, ${ }^{31}$ participants wore actigraphs (ActiGraph GT9X) on their non-dominant wrist 24 hours/day for seven consecutive days at baseline and post-treatment. Data were autoscored through the CentrePoint programme using the validated Cole-Kripke algorithm. ${ }^{34}$ Actigraphy data patterns were then systematically examined for validity and extreme sleep period deviations from diary data. Changes were made to the autoscored major sleep period times based on a standardised procedure developed by our lab ${ }^{33}$; see online supplemental file for details.

\section{Insomnia Severity Index}

This seven-item questionnaire assesses severity of insomnia disorder symptoms. Items are rated from 0 to 4 , scores range from 0 to 28 , and scores are interpreted as follows: 8-14=subthreshold insomnia, 15-21=moderate clinical insomnia and 22-28=severe clinical insomnia. Herein, participants who scored $\geq 8$ on the ISI combined with $\mathrm{SOL}>30 \mathrm{~min}$ and/or spending WASO >30 min were considered part of the insomnia group. ${ }^{35}$

\section{Pittsburgh Sleep Quality Index}

This 10-item questionnaire assesses sleep habits during the past month. Four questions prompt open-ended responses about the time the participant goes to bed, SOL, time out of bed and TST. The remaining items are rated from 0 (not during the past month) to 3 (three or more times a week). Scores range from 0 to 21 , with scores $>5$ indicating poor sleep quality. ${ }^{36}$

\section{Dysfunctional Beliefs and Attitudes about Sleep}

This 16-item questionnaire assesses sleep-related cognitions that may be important for the development and maintenance of insomnia. Items are rated from 0 (strongly disagree) to 10 (strongly agree). Higher scores indicate more dysfunctional beliefs. ${ }^{37}$

\section{Disease activity}

Patient-reported outcomes-3 (PRO-3) ${ }^{38}$ items were used to assess disease activity for both $\mathrm{UC}$ and $\mathrm{CD}$. At baseline, participants rated their stool frequency, abdominal pain, rectal bleeding and general well-being on average over the past 7 days. Participants also answered these questions in the evening diary about the past 1 day. To keep measurement consistent across $\mathrm{CD}$ and UC, we categorised participants as having active or inactive disease at 
baseline and post-treatment. Participants with UC were classified based on a previously established scoring algorithm. ${ }^{39}$ For $\mathrm{CD}$, we created standardised rules to identify participants who were not currently in remission ('active' disease). Participants with CD who were categorised as active included those who endorsed: (1) at least one more loose stool than normal per day, mild abdominal pain or worse, and feeling slightly under par or worse, (2) at least moderate pain and either one or more loose stools than normal per day or feeling slightly under par or worse, (3) five or more loose stools than normal per day and either mild abdominal pain or worse or feeling slightly under par or worse, (4) severe abdominal pain or (5) feeling generally poor or worse.

\section{Qualitative assessment}

After the 1-month follow-up assessment, a study investigator conducted a semi-structured phone interview to elicit feedback on participants' experiences in the trial, including any difficulties with study procedures and/ or the intervention. The interview guide also explored participants' views on treating sleep as part of IBD care. Data collected during the interview were recorded through field notes and audio recordings and were analysed using thematic analysis. ${ }^{40}$ One member of the research team conducted descriptive coding, including reading through field notes, listening to audio recordings and coding responses according to a topic. Codes were then sorted into themes that related to experiences in the trial and views on treating sleep as part of IBD. Themes were generated inductively. A second member of the research team reviewed the coded data and themes to confirm that they were represented in the data and formed a coherent pattern.

\section{Sample size}

Based on widely cited guidance on the purpose of pilot feasibility studies, ${ }^{41}$ a sample size of 10 was determined to be sufficient to investigate feasibility and acceptability of CBT-I, to develop protocols for recruitment and retention for a larger randomised controlled trial (RCT), and to investigate preliminary evidence of effectiveness of CBT-I. This sample size is comparable to similar pilot trials, which included 8-13 participants per trial arm. ${ }^{42-44}$ Feasibility results do not necessarily generalise beyond the inclusion and exclusion criteria of the pilot design.

\section{Data analytical strategy}

Initial analyses focused on comparisons between participants with and without insomnia in order to better characterise our intervention sample. For participants who proceeded to intervention, feasibility data were reported using descriptive statistics. Other outcome data were examined using paired t-tests and Pearson correlations and alpha was set at $p<0.05$. Effect sizes (Hedge's $g$ ) were used to examine the magnitude of change from pre- to post-treatment. Hedge's g was interpreted as $\geq 0.2=$ small, $>0.5=$ medium and $\geq 0.8=$ large ${ }^{45}$

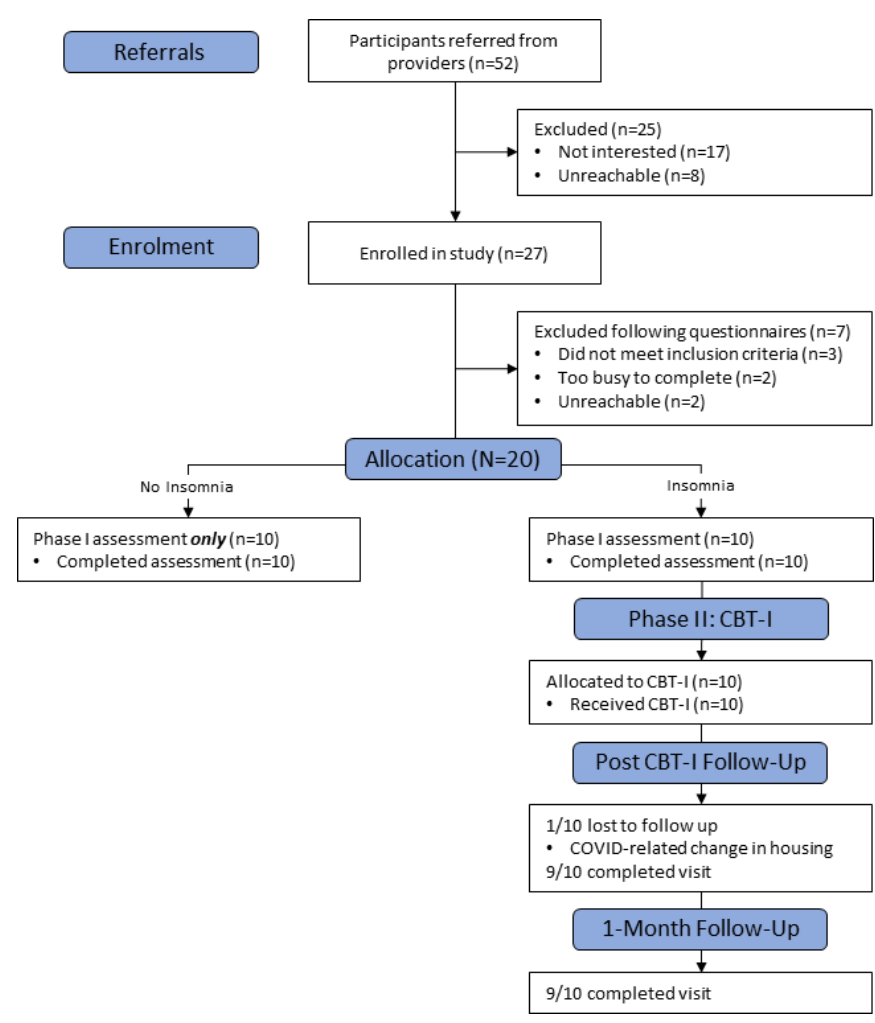

Figure 1 CONSORT (Consolidated Standards of Reporting Trials) diagram. Proceeding to phase II: CBT-I was based on Insomnia Severity Index score. CBT-I, cognitive-behavioural therapy for insomnia.

\section{RESULTS}

Fifty-two patients were referred to the study and 27 enrolled. Enrolment pre-COVID was $42.3 \%$ and enrolment increased to $61.5 \%$ after the study transitioned to operating fully remotely. Of the 27 individuals who were assessed for eligibility for phase I based on questionnaires, 20 proceeded to the full assessment. As is displayed in the CONSORT (Consolidated Standards of Reporting Trials) diagram (figure 1), participants did not proceed to assessment due to not meeting study criteria, being too busy to participate or being unreachable. Of the 20 participants who completed the full assessment, 10 were considered to have significant insomnia symptoms. Participants with insomnia were invited to participate in phase II, CBT-I intervention. Table 2 bdescribes baseline demographic and clinical characteristics for all participants.

\section{Outcomes}

Phase l: sleep assessment outcomes

Participants with and without insomnia symptoms were not significantly different based on age, race, IBD type, years since IBD diagnosis, number taking IBD medications, number taking steroids, number taking psychiatric medications (including hypnotics) or self-reported disease activity. However, there were significantly more women in the intervention group compared with the assessment only group (90\% vs 20\%, respectively); Fisher's exact test $\mathrm{p}=0.002$. As expected, the intervention group (insomnia) also scored significantly worse across measures of sleep as 
Table 2 Participant demographics

\begin{tabular}{|c|c|c|c|}
\hline Demographic variable & $\begin{array}{l}\text { Insomnia: } \\
\text { mean (M), SD and/or no reporting }\end{array}$ & $\begin{array}{l}\text { No insomnia: } \\
\text { mean (M), SD and/or no reporting }\end{array}$ & $\begin{array}{l}\text { Significance test for } \\
\text { group differences }\end{array}$ \\
\hline Age & $\begin{array}{l}\mathrm{M}=42.70, \mathrm{SD}=16.30 \text { (range }=23-67 \\
\text { years) }\end{array}$ & $\begin{array}{l}M=48.00, S D=15.58 \text { (range }=29-73 \\
\text { years) }\end{array}$ & $t(18)=0.74, p=0.47$ \\
\hline Sex & 9 female, 1 male & 2 female, 8 male & Fisher's exact $p=0.002$ \\
\hline Race/ethnicity & 10 white non-Hispanic & 10 white non-Hispanic & Fisher's exact $p=1.0$ \\
\hline IBD type and location & $\begin{array}{l}7 \text { Crohn's disease } \\
\text { Location } \\
3 \text { ileal only } \\
2 \text { ileocolonic } \\
1 \text { colonic only } \\
1 \text { upper tract, colonic } \\
\text { Phenotype } \\
3 \text { inflammatory } \\
3 \text { stricturing } \\
1 \text { penetrating } \\
\text { Perianal Involvement } \\
3 \text { yes } \\
4 \text { no } \\
3 \text { ulcerative colitis } \\
3 \text { left sided disease }\end{array}$ & $\begin{array}{l}5 \text { Crohn's disease } \\
3 \text { ileal only } \\
1 \text { ileocolonic } \\
1 \text { colonic only } \\
\text { Phenotype } \\
1 \text { inflammatory } \\
3 \text { stricturing } \\
1 \text { penetrating } \\
\text { Perianal Involvement } \\
2 \text { yes } \\
3 \text { no } \\
5 \text { ulcerative colitis } \\
3 \text { left sided } \\
2 \text { extensive colitis }\end{array}$ & $\begin{array}{l}\text { Fisher's exact for } \\
\text { Crohn's vs UC } p=0.65\end{array}$ \\
\hline $\begin{array}{l}\text { Years since IBD } \\
\text { diagnosis }\end{array}$ & $\begin{array}{l}M=14.50, S D=12.87 \text { (range }=1-44 \\
\text { years) }\end{array}$ & $\begin{array}{l}M=11.80, S D=9.39(\text { range }=2-30 \\
\text { years) }\end{array}$ & $t(18)=-.56, p=0.58$ \\
\hline IBD medications* & $\begin{array}{l}9 \text { taking: } \\
3 \text { infliximab alone } \\
2 \text { vedolizumab alone } \\
1 \text { ustekinumab alone } \\
1 \text { ustekinumab+prednisone } \\
1 \text { adalimumab +prednisone } \\
1 \text { mesalamine alone }\end{array}$ & $\begin{array}{l}10 \text { taking: } \\
2 \text { adalimumab alone } \\
1 \text { adalimumab +azathioprine } \\
1 \text { infliximab alone alone } \\
1 \text { infliximab+prednisone } \\
1 \text { ustekinumab+prednisone } \\
1 \text { vedolizumab alone } \\
1 \text { mesalamine alone } \\
1 \text { mesalamine +methotrexate } \\
1 \text { sulfasalazine alone }\end{array}$ & Fisher's exact $p=1.0$ \\
\hline $\begin{array}{l}\text { Psychiatric medications } \\
\text { (including sleep } \\
\text { medications and } \\
\text { hypnotics)* }^{*}\end{array}$ & $\begin{array}{l}3 \text { taking: } \\
2 \text { antidepressants } \\
1 \text { antidepressant, anticonvulsant and } \\
\text { atypical antipsychotic }\end{array}$ & 0 taking & Fisher's exact $p=0.21$ \\
\hline $\begin{array}{l}\text { Disease status (remission } \\
\text { vs active) based on } \\
\text { PRO-3 }\end{array}$ & $\begin{array}{l}4 \text { in remission } \\
6 \text { active disease }\end{array}$ & $\begin{array}{l}5 \text { in remission } \\
5 \text { active disease }\end{array}$ & $\begin{array}{l}X^{2}(1, N=20)=0.20 \\
p=0.65\end{array}$ \\
\hline
\end{tabular}

Bolded values are significant though should be interpreted with caution given our small sample size.

${ }^{*}$ Dose did not change over the course of the trial.

IBD, inflammatory bowel disease; PRO-3, patient-reported outcome-3; UC, ulcerative colitis.

compared with the participants without insomnia (table 3). Participants with insomnia reported worse sleep continuity via daily diary and questionnaires; only sleep duration did not differ significantly between the two groups (insomnia $=7.21$ hours/night, no insomnia $=7.30$ hours/ night). As is shown in table 4, actigraphy data showed a similar pattern of results. WASO was significantly greater in participants with insomnia (117.78 vs $59.47 \mathrm{~min}$ ), while TST and SE were not significantly different.

Phase II: pilot feasibility trial outcomes

Feasibility

Of the 10 participants invited to enrol in CBT-I, $100 \%$ elected to proceed with intervention, $100 \%$ completed all 5 CBT-I sessions and $100 \%$ completed all 3 phone calls. Retention through the last CBT-I session was $100 \%$ $(\mathrm{N}=10)$, and retention through the post-CBT-I assessment and 1-month follow-up assessments was 90\% ( $\mathrm{N}=9$ ). Post-CBT-I, 1 participant dropped out due to COVID19-related housing and job changes. Participants also reported CBT-I to be highly acceptable. Following session 1 , the treatment acceptability questionnaire mean $=3.78$ ( $\mathrm{SD}=0.32),>1.5 \mathrm{SD}$ above the published mean. Sleep diary completion rate was $99.6 \%$ during phase I (2 weeks) and $98.8 \%$ during phase II ( 7 weeks). During the 1-week follow-up, we received complete diaries for $8 / 10$ people; those 8 participants evidenced a completion rate 
Table 3 Comparisons between participants with and without insomnia symptoms and preliminary evidence of efficacy of CBT-I based on daily diary data and questionnaires

\begin{tabular}{|c|c|c|c|c|c|c|c|}
\hline & $\begin{array}{l}\text { No insomnia } \\
(\mathrm{N}=10)^{\star} \\
\text { Mean (SE) }\end{array}$ & $\begin{array}{l}\text { Pre-CBT-I } \\
(\mathrm{N}=10) \\
\text { Mean (SE) }\end{array}$ & $\begin{array}{l}\text { Insomnia vs } \\
\text { no insomnia } \\
t \text { test and } \\
\text { effect size } \\
(95 \% \mathrm{Cl})\end{array}$ & $\begin{array}{l}\text { Immediately } \\
\text { post-CBT-I } \\
(\mathrm{N}=10) \dagger \ddagger\end{array}$ & $\begin{array}{l}\text { Insomnia } \\
\text { pretreatment vs } \\
\text { post-treatment } \\
T \text { test and effect } \\
\text { size }(95 \% \mathrm{Cl})\end{array}$ & $\begin{array}{l}1 \text { month } \\
\text { follow-up } \\
\text { ( } N=8) \S \\
\text { Mean (SE) }\end{array}$ & $\begin{array}{l}\text { Insomnia } \\
\text { pretreatment vs } \\
\text { follow-up T test } \\
\text { and effect size } \\
(95 \% \mathrm{Cl})\end{array}$ \\
\hline \multicolumn{8}{|c|}{ Primary CBT-I outcomes } \\
\hline $\begin{array}{l}\text { Sleep-onset } \\
\text { latency in } \\
\text { minutes }\end{array}$ & $12.52(2.80)$ & $47.40(9.02)$ & $\begin{array}{l}t(16)=3.34 \\
p=0.004 ; 1.51 \\
(.47 \text { to } 2.52)\end{array}$ & $17.11(3.93)$ & $\begin{array}{l}t(9)=3.95 \\
p=0.003 ; 1.25 \\
(.39 \text { to } 2.07)\end{array}$ & $23.03(7.60)$ & $\begin{array}{l}t(7)=2.33 \\
p=0.05 ; .82 \\
(-0.01 \text { to } 1.53)\end{array}$ \\
\hline $\begin{array}{l}\text { Wake after } \\
\text { sleep onset in } \\
\text { minutes }\end{array}$ & 16.35 (5.19) & $36.41(3.12)$ & $\begin{array}{l}t(16)=3.46 \\
p=0.003 ; 1.56 \\
(.51 \text { to } 2.58)\end{array}$ & $17.68(4.75)$ & $\begin{array}{l}t(9)=3.86 \\
p=0.004 ; 1.17 \\
(.36 \text { to } 1.95)\end{array}$ & $21.14(5.55)$ & $\begin{array}{l}t(7)=2.92 \\
p=0.02 ; .98(.13 \\
\text { to } 1.78)\end{array}$ \\
\hline Hours of sleep & $7.30(.12)$ & $7.21(.78)$ & $\begin{array}{l}t(16)=-.09 \\
p=0.93 ;-0.04 \\
(-0.93-.89)\end{array}$ & $7.52(.22)$ & $\begin{array}{l}t(9)=-.37, p=0.72 \\
-0.11(-0.70-.49)\end{array}$ & $7.27(.18)$ & $\begin{array}{l}t(7)=-.71 \\
p=0.50 ;-0.24 \\
(-0.89-.44)\end{array}$ \\
\hline $\begin{array}{l}\text { Insomnia } \\
\text { Severity Index }\end{array}$ & $5.40(.64)$ & $16.50(1.68)$ & $\begin{array}{l}t(18)=6.17 \\
p<0.001 ; 2.64 \\
(1.43 \text { to } 3.83)\end{array}$ & $4.38(.65)$ & $\begin{array}{l}t(7)=8.79, \\
p<0.001 ; 2.94 \\
(1.29 \text { to } 4.57)\end{array}$ & $4.86(1.39)$ & $\begin{array}{l}t(6)=6.97 \\
p<0.001 ; 2.47 \\
(.93 \text { to } 3.98)\end{array}$ \\
\hline $\begin{array}{l}\text { Pittsburg sleep } \\
\text { quality index }\end{array}$ & $5.40(.58)$ & $10.90(1.03)$ & $\begin{array}{l}t(18)=4.66 \\
p<0.001 ; 2.00 \\
(.92 \text { to } 3.04)\end{array}$ & $4.50(1.10)$ & $\begin{array}{l}t(7)=5.53 \\
p=0.001 ; 1.85 \\
(.68 \text { to } 2.98)\end{array}$ & $4.43(.84)$ & $\begin{array}{l}t(6)=8.29 \\
p<0.001 ; 2.93 \\
(1.17 \text { to } 4.68)\end{array}$ \\
\hline \multicolumn{8}{|c|}{ Secondary CBT-I outcomes } \\
\hline $\begin{array}{l}\text { Dysfunctional } \\
\text { beliefs and } \\
\text { attitudes about } \\
\text { sleep }\end{array}$ & $3.26(.33)$ & $6.18(.42)$ & $\begin{array}{l}t(18)=5.52, \\
p<0.001 ; 2.37 \\
(1.21 \text { to } 3.48)\end{array}$ & $3.24(.33)$ & $\begin{array}{l}t(7)=7.18, \\
p<0.001 ; 2.40 \\
(.99 \text { to } 3.78)\end{array}$ & $3.11(.59)$ & $\begin{array}{l}t(6)=7.43 \\
p<0.001 ; 2.63 \\
(1.01 \text { to } 4.23)\end{array}$ \\
\hline
\end{tabular}

Bolded values are significant, though should be interpreted with caution given our small sample size.

${ }^{*} n=8$ participants had complete diary data.

†The last week of CBT-I treatment data were used for each participant in data analyses.

$\neq n=8$ participants had questionnaire data.

$\S \mathrm{n}=7$ participants had questionnaire data.

CBT-I, cognitive-behavioural therapy for insomnia; PRO-3, patient-reported outcome-3.

Table 4 Comparisons between participants with and without insomnia symptoms and preliminary evidence of efficacy of CBT-I based on actigraphy data

\begin{tabular}{|c|c|c|c|c|c|}
\hline & $\begin{array}{l}\text { No insomnia } \\
(\mathrm{N}=9) \\
\text { Mean (SE) }\end{array}$ & $\begin{array}{l}\text { Pre-CBT-I } \\
(\mathrm{N}=8) \\
\text { Mean (SE) }\end{array}$ & $\begin{array}{l}\text { Insomnia vs no } \\
\text { insomnia } \\
\text { t test and effect } \\
\text { size }(95 \% \mathrm{Cl}) \\
\end{array}$ & $\begin{array}{l}\text { 1-month follow-up } \\
(\mathrm{N}=7)\end{array}$ & $\begin{array}{l}\text { Insomnia } \\
\text { pretreatment vs post- } \\
\text { treatment T test and } \\
\text { effect size }(95 \% \mathrm{Cl})\end{array}$ \\
\hline $\begin{array}{l}\text { Wake after sleep onset } \\
\text { in minutes }\end{array}$ & $59.47(10.58)$ & 117.78 (23.99) & $\begin{array}{l}t(15)=2.32 \\
p=0.035 ; 1.07 \\
(0.07 \text { to } 2.03)\end{array}$ & $61.84(11.83)$ & $\begin{array}{l}t(6)=4.13, p=0.006 ; \\
1.46(0.38 \text { to } 2.50)\end{array}$ \\
\hline Hours of sleep & $6.59(.24)$ & $7.16(.29)$ & $\begin{array}{l}t(15)=1.51, p=0.15 \\
0.70(-0.25 \text { to } \\
1.62)\end{array}$ & $6.52(0.31)$ & $\begin{array}{l}t(6)=5.79, p=0.001 \\
2.05(0.70 \text { to } 3.35)\end{array}$ \\
\hline Sleep efficiency (\%) & $86.5 \%(2.45)$ & $78.6 \%(3.55)$ & $\begin{array}{l}t(15)=-1.87 \\
p=0.08 ;-0.86 \\
(-1.81 \text { to } 0.10)\end{array}$ & $86.3 \%(2.24)$ & $\begin{array}{l}t(6)=-2.64, p=0.039 \\
0.93(0.05 \text { to } 1.77)\end{array}$ \\
\hline
\end{tabular}

Bolded values are significant though should be interpreted with caution given our small sample size.

CBT-I, cognitive-behavioural therapy for insomnia. 
of $100 \%$. For the two participants with missing data, one dropped out of the trial after intervention and the other was not asked to complete the diary due to a protocol execution error. Finally, 95\% of participants (19/20) completed at least 5 days of actigraphy during the assessment phase and 80\% (16/20) completed all 7 days. Data from two participants who completed 7 days of actigraphy were not correctly exported into the CentrePoint data management programme due to a protocol execution error. During the follow-up assessment, 100\% (9/9) participants completed at least 5 days of actigraphy and $66.6 \%(5 / 9)$ completed 7 days.

\section{Preliminary evidence of efficacy}

As is shown in table 3, paired-samples t-tests indicated that participants experienced significant improvement in SOL, WASO and SE over the course of the trial. T-tests revealed large within-subject effect sizes (1.14-1.25). TST did not change statistically significantly $(\mathrm{p}=0.72)$, though increased by an average of $18.6 \mathrm{~min}$ from baseline to post-CBT-I. Participants also experienced significant decreases in their ISI and PSQI scores, with large effect sizes for improvement (1.85-2.94). All of the significant changes from pre-CBT-I to post-CBT-I remained significant when controlling for multiple comparisons using a Holm-Bonferroni correction. Further, improvements in sleep were maintained 1 month after CBT-I.

As is shown in table 4, actigraphy data were mostly consistent with the daily diary. Post CBT-I, intervention participants had significantly reduced WASO and significantly improved SE However, they also had significantly reduced TST. Of note, the actigraphy data post CBT-I was comparable to the data from the participants without insomnia.

\section{Exploratory outcomes}

Similar to other sleep measures, scores on the DBAS improved significantly during CBT-I $(g=2.40)$. Additionally, the percentage of participants reporting active IBD changed significantly over the course of the trial. Pre-CBT-I, $60 \%$ of participants reported active disease (compared with $50 \%$ of participants without insomnia). Immediately post-CBT-I, none of the intervention participants reported active disease $(0 \%)$, McNeman's test $\mathrm{p}=0.03$. At the 1 -month follow-up, one of eight participants $(12.5 \%)$ reported active disease. These changes in disease activity occurred without any changes in IBD medications throughout the trial, aside from one participant who changed from rifaximin to mesalamine.

\section{Qualitative data}

Nine of 10 participants completed the post-trial phone call and overall, the qualitative data supported the feasibility and acceptability of CBT-I in IBD. Data, codes, and themes were explored by two of the authors, and the two coders were in agreement about the codes and themes. Primarily, all participants shared that they found the intervention worthwhile, felt like the treatment met or exceeded their needs, and reported they would definitely recommend the treatment to a friend. For example, 'It definitely exceeded what I expected. I remember the first visit, thinking this is never going to work. And I was really surprised at how quickly I was able to make it work and actually see and feel the results.'

Across interviews, several themes related to the acceptability of the intervention and relevant facilitators emerged. First, participants generally liked the use of telehealth for the intervention, though it should be noted that as much of the study took place during the COVID-19 pandemic, during which telehealth has been more common for medical visits. For example, participants stated:

I think it was fine. I mean, I'm kind of used to it because that's how we're doing a lot of things. Having not done it face-to-face I don't know that I missed out on anything, but I think you still get the same information and experience out of it.

For me, it was so much easier, and this was the perfect type of visit to do over a computer.

Participants also appreciated both the structure of the intervention, including the spacing of the visits and the phone calls, and the content, particularly how it specifically related to IBD. Comments about the intervention structure include:

The spacing of them [the treatment sessions] was good, meeting every other week and the phone calls to check in, it was all really good.

The study was long, but it made sense that it was long and it was helpful. The session tapering [going from weekly to every other week] and calls in-between were all helpful.

Comments about the intervention content include:

To know this was specifically geared toward IBD was just incredibly helpful. Because it's for IBD, you [the interventionist \& study team] know what we're going through, and that we're all very different in what we're going through, and also have some similar experiences.

My favorite part was our final meeting, where we talked about how life happens, and you might not be able to keep up with this all the time, but here is how you can get back on track when you get off the track. That was so extremely helpful. You understand I'm a human being that has a life.

Some of them [treatment activities] I still use. Subconsciously you just start thinking about the activities we did do and you use them. I use the relaxation and the setting aside time for worrying.

Finally, participants indicated that they really appreciated the ongoing access to the study team.

I knew that the interventionist and research coordinator were available if I had questions all 
I had to do was send a note and I got a response immediately.

With regard to treatment barriers, the only clear theme that emerged was difficulty with or dislike of the daily diary. However, participants generally found that while they did not like the daily diaries, they were overall helpful. For example:

Sometimes remembering to actually log into the tracker was a big one, but it forces you to slow down a little when you're actually looking at it, because it does make sense to see it.

I think the actual filling out the charts every day, even though it was a little bit tedious, to be able to go back and look over them, was really helpful. Because it is easy to be like oh, it's not much different than it was a week ago. But having it be easy, just a click click, made it easier to keep up with.

Participants were also asked what they thought about treating sleep as part of IBD care and several themes emerged. Primarily, participants indicated that treating sleep was very helpful or important and were overall quite enthusiastic about the intervention. For example: 'It was extremely relevant,' 'I thought it was great,' and 'It's been really helpful for me.'

While some participants were already aware of the connection between their sleep and IBD symptoms, others indicated that it had never occurred to them that sleep and IBD could be related. A participant who was already aware of the sleep/IBD connection shared:

I think it completely correlates to potential flare ups because I do feel like my gut is associated with my sleep.

Examples from participants for whom the sleep/IBD relationship was a novel idea include:

It was an unexpected avenue to take but one of the best things I have done in a long time,

I know sleep in general is important, but I don't think I would have thought of it initially as a treatment to help with IBD, and

It met a need I didn't know was a need.

Finally, the last theme to emerge was that treating insomnia had impacts on health beyond the specific improvements in sleep. Participants indicated:

\section{Overall I feel better,}

Things are more consistent for me now...I'm more rested so I can cope better. Even on a bad day I can cope. Things are more manageable, and

By attacking the sleep issue, it started to make a healthy interrelationship between the three issues [sleep, IBD, and mental health] in a really great way. Improvements in all three for sure
Harms

No adverse events were reported throughout this trial.

\section{DISCUSSION}

In this trial, we sought to assess sleep patterns in people with mild-to-moderately active IBD and provide treatment to participants with insomnia symptoms. As expected, during the assessment phase of the trial participants with insomnia evidenced significantly worse sleep than those without insomnia based on actigraphy, daily diary and questionnaires.

During the intervention phase, we found impressive support for the feasibility of CBT-I in people with IBD. Specifically, despite the burden of completing daily diaries for a total of $9+$ weeks, completion rates were very high $(>98 \%)$. Similarly, $95 \%$ of participants completed actigraphy during the assessment phase and $100 \%$ completed it postintervention. Postintervention qualitative data also indicated that participants liked the use of telehealth for the study, the intervention structure and content, and the ease of access to the interventionist and study team. Additionally, while many participants were surprised that there was a connection between sleep and IBD, they all believed the intervention was worthwhile. These statements corroborate our low attrition rate-all participants with insomnia symptoms elected to complete CBT-I and all of them completed 5/5 intervention visits. Further, many participants indicated that once they were sleeping better, they began to feel physically and mentally better in other ways. It is also important to note that while we used a low insomnia severity cut-off for inclusion (ISI $\geq 8$ combined with SOL or WASO $>30 \mathrm{~min}$ ) to ensure recruitment was feasible, all baseline ISI scores in the insomnia groups were 11 or greater, and thus above the threshold for clinical insomnia. ${ }^{46}$

Findings also supported the preliminary evidence of efficacy of CBT-I. From preintervention to postintervention, participants who received CBT-I improved significantly in their diary SOL, WASO and SE, and questionnaire based insomnia and sleep quality; these gains were maintained at the 1-month follow-up assessment. The percentage of participants with active disease based on the PRO-3 improved significantly over the course of the intervention, from $60 \%$ pre- CBT-I to $0 \%$ immediately post and $12.5 \%(1 / 8) 1$ month later without significant changes in IBD medications.

Overall, our diary data are consistent with other research on CBT-I outcomes. Recent meta-analyses of the effects of CBT-I in primary and chronic insomnia ${ }^{2447}$ demonstrated a 19-20 min decrease in diary SOL, 26-40 min decrease in diary WASO, and 10\%-26\% increase in diary SE. Our results are also consistent a 2021 metaanalysis of behavioural and psychological treatments for chronic insomnia. ${ }^{28}$ Actigraphy data were also consistent with the extant literature. We found a significant decrease in WASO and increase in SE; however, participants also experienced a significant decrease in TST. 
Unfortunately, this decrease in TST is a common consequence of the sleep restriction component of CBTI, ${ }^{48}$ which prioritises sleep quality over quantity in the short term. CBT-I studies with longer follow-up periods typically show that TST eventually returns to baseline levels. $^{49}$

Additionally, Hashash et al recently examined a brief behavioural treatment for sleep disturbance (BBTS) in adolescents and young adults with IBD. ${ }^{50}$ Participants who scored $>5$ on the PSQI participated in a 4-week insomnia-based treatment, following which participants with persistent fatigue were offered bupropion. Similar to our findings, following BBTS participants evidenced improvements in sleep. While our study differs from that by Hashash and colleagues in a number of ways, one important methodological difference is in participant selection. Elevations on the PSQI are reflective of a heterogeneous group of sleep disorders, and thus it is possible that participants who received BBTS had sleep problems other than insomnia, which would not have been as responsive to the intervention. Additionally, a significant and unique strength of our study involves the inclusion of pain-specific content in the CBT-I intervention protocol.

\section{Limitations and future research}

As this was a small, uncontrolled pilot study, it is impossible to determine whether improvements in insomnia were due to CBT-I, as opposed to other factors such as natural improvement in symptoms or the therapeutic alliance. Further, based on this small, homogeneous sample, it is unknown at present whether our results will generalise to larger samples. As this study has demonstrated feasibility and preliminary evidence of efficacy of CBT-I, a natural and necessary next step would involve efficacy testing within a pilot RCT. ${ }^{51}$

An additional limitation was our reliance on PRO-3 of disease activity. While PROs are becoming a standard clinical trial outcome for IBD, ${ }^{52}$ objective markers of disease activity would provide greater insight into possible impact of CBT-I. Further, poor sleep may be related to elevated faecal calprotectin in people with $\mathrm{CD}^{53}$ and emerging literature in other populations has demonstrated improvements in C-reactive protein following CBT-I. ${ }^{54} 55$ Thus, future research aimed at assessing the physiological impacts of insomnia treatment would likely contribute significantly to our understanding of disease processes in IBD.

Finally, this study did not use polysomnography (PSG) or similar testing to physiologically screen for sleep apnea or other sleep disorders. Although these disorders were excluded based on questionnaires, it remains possible that some participants had occult sleep disorders in addition to insomnia. Future studies should include PSG sleep assessment to ascertain whether these potential comorbidities influence CBT-I outcomes in IBD.

\section{Clinical implications}

As we have demonstrated elsewhere, people with active IBD experience significant IBD-related sleep disruptions (eg, night-time awakenings for bowel movements, pain that interferes with sleep) ${ }^{16}$ However, our findings indicate that CBT-I can still be effective, with improvements that are similar to other CBT-I trials. Further, qualitative data suggested that many participants were surprised to learn about the association between sleep and IBD and would not have thought that insomnia treatment could impact other areas of functioning. Thus, the onus to ask about sleep problems and suggest treatments may rely on the gastroenterologist or others involved in IBD care. Elsewhere we have suggested questions that providers could ask patients about their sleep and ways to respond to sleep problems that are consistent with a CBT-I model.$^{56}$ Importantly, these suggestions go beyond the scope of sleep hygiene, which is not effective for addressing chronic insomnia on its own. ${ }^{28}$ Minimally, providers could ask patients how their sleep is, and if a patient indicates that there are significant problems, refer out for further assessment or treatment. If patients specifically report problems with insomnia, providers could consider referral to a psychologist, behavioural sleep medicine specialist, or evidence-based online programme (eg, Sleepio.com, Somryst).

\section{CONCLUSIONS}

Overall, these preliminary data highlight the potential impact that CBT-I can have in people with IBD. Providers are encouraged to ask their patients about insomnia or other sleep concerns and give evidence-based suggestions and/or specialist referrals. Future research should seek to expand on the current findings, in addition to determining the other ways in which treatment of insomnia can impact IBD symptoms and severity.

Contributors All authors included in this paper have contributed to the manuscript. JS-D, MTS, KAA and CS designed the study. JS-D and BCS served as study interventionists and HGH served as study coordinator. JS-D and BCS conducted study analyses and JS-D wrote the first draft of the manuscript. All authors contributed to the interpretation of data, critically reviewed the manuscript for intellectual content, and approved the final version. JS-D is responsible for the overall content as guarantor.

Funding Gary Tucker Junior Investigator Research Award (JS-D); Scholarship Enhancement in Academic Medicine Award (JS-D).

Competing interests None declared.

Patient consent for publication Not applicable.

Ethics approval This study and procedures were approved by the DartmouthHitchcock Health Human Research Protection Programme (IRB; \#02000051).

Provenance and peer review Not commissioned; externally peer reviewed. Data availability statement Data are available on reasonable request.

Supplemental material This content has been supplied by the author(s). It has not been vetted by BMJ Publishing Group Limited (BMJ) and may not have been peer-reviewed. Any opinions or recommendations discussed are solely those of the author(s) and are not endorsed by BMJ. BMJ disclaims all liability and responsibility arising from any reliance placed on the content. Where the content includes any translated material, BMJ does not warrant the accuracy and reliability of the translations (including but not limited to local regulations, clinical guidelines, 
terminology, drug names and drug dosages), and is not responsible for any error and/or omissions arising from translation and adaptation or otherwise.

Open access This is an open access article distributed in accordance with the Creative Commons Attribution Non Commercial (CC BY-NC 4.0) license, which permits others to distribute, remix, adapt, build upon this work non-commercially, and license their derivative works on different terms, provided the original work is properly cited, appropriate credit is given, any changes made indicated, and the use is non-commercial. See: http://creativecommons.org/licenses/by-nc/4.0/.

\section{ORCID iD}

Jessica K Salwen-Deremer http://orcid.org/0000-0001-6498-3729

\section{REFERENCES}

1 Ananthakrishnan AN, Long MD, Martin CF, et al. Sleep disturbance and risk of active disease in patients with Crohn's disease and ulcerative colitis. Clin Gastroenterol Hepatol 2013;11:965-71.

2 Ali T, Madhoun MF, Orr WC, et al. Assessment of the relationship between quality of sleep and disease activity in inflammatory bowel disease patients. Inflamm Bowel Dis 2013;19:2440-3.

3 Ranjbaran Z, Keefer L, Farhadi A, et al. Impact of sleep disturbances in inflammatory bowel disease. J Gastroenterol Hepatol 2007;22:1748-53.

4 Uemura R, Fujiwara Y, Iwakura N, et al. Sleep disturbances in Japanese patients with inflammatory bowel disease and their impact on disease flare. Springerplus 2016;5:5.

5 Sofia MA, Lipowska AM, Zmeter N, et al. Poor sleep quality in Crohn's disease is associated with disease activity and risk for hospitalization or surgery. Inflamm Bowel Dis 2020;26:1251-9.

6 Graff LA, Vincent N, Walker JR, et al. A population-based study of fatigue and sleep difficulties in inflammatory bowel disease. Inflamm Bowel Dis 2011;17:1882-9.

7 Keefer L, Stepanski EJ, Ranjbaran Z, et al. An initial report of sleep disturbance in inactive inflammatory bowel disease. J Clin Sleep Med 2006;2:409-16.

8 Association AP. Dsm 5: American psychiatric association, 2013

9 Singareddy R, Vgontzas AN, Fernandez-Mendoza J, et al. Risk factors for incident chronic insomnia: a general population prospective study. Sleep Med 2012;13:346-53.

10 Mai E, Buysse DJ. Insomnia: prevalence, impact, pathogenesis, differential diagnosis, and evaluation. Sleep Med Clin 2008;3:167-74.

11 Taylor DJ, Mallory LJ, Lichstein KL, et al. Comorbidity of chronic insomnia with medical problems. Sleep 2007;30:213-8.

12 Taylor DJ, Pruiksma KE. Cognitive and behavioural therapy for insomnia (CBT-I) in psychiatric populations: a systematic review. Int Rev Psychiatry 2014;26:205-13.

13 Smith MT, Perlis ML, Smith MS, et al. Sleep quality and presleep arousal in chronic pain. J Behav Med 2000;23:1-13

14 Morin CM, Gibson D, Wade J. Self-Reported sleep and mood disturbance in chronic pain patients. Clin J Pain 1998;14:311-4.

15 Scott AJ, Flowers O, Rowse G. Do specific types of sleep disturbances represent risk factors for poorer health-related quality of life in inflammatory bowel disease? A longitudinal cohort study. $\mathrm{Br}$ $J$ Health Psychol 2021;26:90-108.

16 Salwen-Deremer JK, Smith MT, Haskell HG, et al. Poor sleep in inflammatory bowel disease is reflective of distinct sleep disorders. Dig Dis Sci 2021. doi:10.1007/s10620-021-07176-y. [Epub ahead of print: 30 Jul 2021].

17 Spielman AJ, Caruso LS, Glovinsky PB. A behavioral perspective on insomnia treatment. Psychiatr Clin North Am 1987;10:541-53.

18 Qaseem A, Kansagara D, Forciea MA, et al. Management of chronic insomnia disorder in adults: a clinical practice guideline from the American College of physicians. Ann Intern Med 2016;165:125-33.

19 Jacobs GD, Pace-Schott EF, Stickgold R, et al. Cognitive behavior therapy and pharmacotherapy for insomnia: a randomized controlled trial and direct comparison. Arch Intern Med 2004;164:1888-96.

20 Smith MT, Perlis ML, Park A, et al. Comparative meta-analysis of pharmacotherapy and behavior therapy for persistent insomnia. Am J Psychiatry 2002;159:5-11.

21 Riemann D, Baglioni C, Bassetti C, et al. European guideline for the diagnosis and treatment of insomnia. J Sleep Res 2017;26:675-700.

22 Finan PH, Buenaver LF, Coryell VT, et al. Cognitive-Behavioral therapy for comorbid insomnia and chronic pain. Sleep Med Clin 2014;9:261-74.

23 Smith MT, Huang MI, Manber R. Cognitive behavior therapy for chronic insomnia occurring within the context of medical and psychiatric disorders. Clin Psychol Rev 2005;25:559-92.
24 Trauer JM, Qian MY, Doyle JS, et al. Cognitive behavioral therapy for chronic insomnia: a systematic review and meta-analysis. Ann Intern Med 2015;163:191-204.

25 Irwin MR, Cole JC, Nicassio PM. Comparative meta-analysis of behavioral interventions for insomnia and their efficacy in middleaged adults and in older adults 55+ years of age. Health Psychol 2006;25:3-14.

26 van Straten A, van der Zweerde T, Kleiboer A, et al. Cognitive and behavioral therapies in the treatment of insomnia: a meta-analysis. Sleep Med Rev 2018;38:3-16.

27 Cavanagh RC, Mackey R, Bridges L, et al. The use of digital health technologies to manage insomnia in military populations. $J$ Technol Behav Sci 2020;5:61-9.

28 Edinger JD, Arnedt JT, Bertisch SM, et al. Behavioral and psychological treatments for chronic insomnia disorder in adults: an American Academy of sleep medicine systematic review, meta-analysis, and grade assessment. J Clin Sleep Med 2021;17:263-98.

29 Perlis ML, Jungquist C, Smith MT, et al. Cognitive behavioral treatment of insomnia: a session-by-session guide. Springer Science \& Business Media, 2006

30 Kyle SD, Aquino MRJ, Miller CB, et al. Towards standardisation and improved understanding of sleep restriction therapy for insomnia disorder: a systematic examination of CBT-I trial content. Sleep Med Rev 2015;23:83-8.

31 Buysse DJ, Ancoli-Israel S, Edinger JD, et al. Recommendations for a standard research assessment of insomnia. Sleep 2006;29:1155-73.

32 Carney CE, Buysse DJ, Ancoli-Israel S, et al. The consensus sleep diary: standardizing prospective sleep self-monitoring. Sleep 2012;35:287-302.

33 Ancoli-Israel S, Martin JL, Blackwell T, et al. The SBSM guide to actigraphy monitoring: clinical and research applications. Behav Sleep Med 2015;13 Suppl 1:S4-38.

34 Cole RJ, Kripke DF, Gruen W, et al. Automatic sleep/wake identification from wrist activity. Sleep 1992;15:461-9.

35 Bastien $\mathrm{CH}$, Vallières $\mathrm{A}$, Morin $\mathrm{CM}$. Validation of the insomnia severity index as an outcome measure for insomnia research. Sleep Med 2001;2:297-307.

36 Buysse DJ, Reynolds CF, Monk TH, et al. The Pittsburgh sleep quality index: a new instrument for psychiatric practice and research. Psychiatry Res 1989;28:193-213.

37 Morin $\mathrm{CM}$, Vallières $\mathrm{A}$, Ivers $\mathrm{H}$. Dysfunctional beliefs and attitudes about sleep (DBAS): validation of a brief version (DBAS-16). Sleep 2007;30:1547-54.

38 Cohen ER, Melmed GY. Making a case for patient-reported outcomes in clinical inflammatory bowel disease practice. Clin Gastroenterol Hepatol 2018;16:603-7.

39 Bewtra M, Brensinger CM, Tomov VT, et al. An optimized patientreported ulcerative colitis disease activity measure derived from the Mayo score and the simple clinical colitis activity index. Inflamm Bowel Dis 2014;20:1-8.

40 Braun V, Clarke V. Using thematic analysis in psychology. Qual Res Psychol 2006;3:77-101.

41 Leon AC, Davis LL, Kraemer HC. The role and interpretation of pilot studies in clinical research. J Psychiatr Res 2011;45:626-9.

42 Tew GA, Leighton D, Carpenter R, et al. High-intensity interval training and moderate-intensity continuous training in adults with Crohn's disease: a pilot randomised controlled trial. BMC Gastroenterol 2019;19:1-11.

43 Vogelaar L, Van't Spijker A, Vogelaar T, et al. Solution focused therapy: a promising new tool in the management of fatigue in Crohn's disease patients psychological interventions for the management of fatigue in Crohn's disease. $J$ Crohns Colitis 2011;5:585-91

44 Siengsukon CF, Alshehri M, Williams $\mathrm{C}$, et al. Feasibility and treatment effect of cognitive behavioral therapy for insomnia in individuals with multiple sclerosis: a pilot randomized controlled trial. Mult Scler Relat Disord 2020;40:101958.

45 Fritz CO, Morris PE, Richler JJ. Effect size estimates: current use, calculations, and interpretation. J Exp Psychol Gen 2012;141:2-18.

46 Morin CM, Belleville G, Bélanger L, et al. The insomnia severity index: psychometric indicators to detect insomnia cases and evaluate treatment response. Sleep 2011;34:601-8.

47 Okajima I, Komada Y, INOUE Y. A meta-analysis on the treatment effectiveness of cognitive behavioral therapy for primary insomnia. Sleep Biol Rhythms 2011;9:24-34.

48 Mitchell LJ, Bisdounis L, Ballesio A, et al. The impact of cognitive behavioural therapy for insomnia on objective sleep parameters: a meta-analysis and systematic review. Sleep Med Rev 2019:47:90-102. 
49 Morin CM, Colecchi C, Stone J, et al. Behavioral and pharmacological therapies for late-life insomnia: a randomized controlled trial. JAMA 1999;281:991-9.

50 Hashash JG, Knisely MR, Germain A, et al. Brief Behavioral Therapy and Bupropion for Sleep and Fatigue in Young Adults With Crohn's Disease: An Exploratory Open Trial Study. Clinical Gastroenterology and Hepatology 2022;20:96-104.

51 Onken LS, Carroll KM, Shoham V, et al. Reenvisioning clinical science: unifying the discipline to improve the public health. Clin Psychol Sci 2014;2:22-34.

52 Williet N, Sandborn WJ, Peyrin-Biroulet L. Patient-Reported outcomes as primary end points in clinical trials of inflammatory bowel disease. Clin Gastroenterol Hepatol 2014;12:1246-56.
53 Gîlc-Blanariu G-E, Ștefnescu G, Trifan AV, et al. Sleep impairment and psychological distress among patients with inflammatory bowel Disease-beyond the obvious. J Clin Med 2020;9:2304.

54 Irwin MR, Olmstead R, Carrillo C, et al. Cognitive behavioral therapy vs. tai chi for late life insomnia and inflammatory risk: a randomized controlled comparative efficacy trial. Sleep 2014;37:1543-52.

55 Irwin MR, Olmstead R, Breen EC, et al. Cognitive behavioral therapy and tai chi reverse cellular and genomic markers of inflammation in late-life insomnia: a randomized controlled trial. Biol Psychiatry 2015;78:721-9.

56 Salwen-Deremer JK, Siegel CA, Smith MT. Cognitive behavioral therapy for insomnia: a promising treatment for insomnia, pain, and depression in patients with IBD. Crohns Colitis 360 2020;2:otaa052. 